\title{
Étude de la transmission père-fils des variations saisonnières du diamètre testiculaire et du pourcentage de spermatozoïdes anormaux chez le bélier lle-de-France. 1. Fils nés en février *
}

\author{
G Colas ${ }^{1 * \star}, \mathrm{J}$ Lefèbvre $2, \mathrm{Y}$ Guérin 1 \\ 1 INRA, station de physiologie de la reproduction, 37380 Nouzilly \\ 2 INRA, laboratoire de génétique factorielle, 78350 Jouy-en Josas Cédex, France
}

(Reçu le 22 décembre 1989; accepté le 27 juin 1990)

\begin{abstract}
Résumé - Nous avons voulu vérifier si les variations saisonnières (VS) du diamètre testiculaire (DT) et du pourcentage de spermatozoïdes anormaux (AM) enregistrés sur 5 béliers lle-de-France adultes, non apparentés (ol, II, III, IV, V), se transmettent à leurs descendants ( $n=18,3$ à 4 descendants $/ 0 ; 5$ familles, $F_{1}$ à $F_{5}$ ). Les peres II a $V$ avaient des $V S$ fortes (o-III et IV), faibles (orll) ou intermédiaires $(o \rightarrow V)$. Le $5^{\theta}$ père était peu sensible (production spermatique) aux variations de la photopériode artificielle et doué d'un pouvoir fécondant élevé au printemps. Les 18 fils ont été contrôlés $(D T$ et $A M)$ entre 8,5 et 46 mois pendant 7 périodes $\left(P_{1}\right.$ à $\left.P_{7}\right)$. Le $D T$ moyen est supérieur chez $F_{1}$ et $F_{2}$, inférieur chez $F_{3}$ et $F_{4}$ à celui de la moyenne générale $(M G)$. $F_{3}$ et $F_{4}$ sont aussi plus proches de la $M G$ en automne qu'au printemps. Les distances entre $F_{1}, F_{2}$ et $F_{3}$ sont toujours significatives. Elles sont minimales entre $F_{3}$ et $F_{4} . F_{5}$ a des valeurs intermédiaires : elles augmentent au printemps et régressent en automne. Les différences entre familles et les valeurs des tests $F$ sont plus faibles pour les $A M$ que pour le $D T$. Dans l'ensemble, $F_{1}$ est au-dessous de $M G, F_{3}$ au-dessus. $F_{2}, F_{4}$ et $F_{5}$ fluctuent autour de cette moyenne. Cette étude montre que les variations saisonnières du diamètre testiculaire et des anomalies morphologiques se transmettent à la descendance chez la race lle-deFrance mais avec plus de précision et d'intensité dans le cas du diamètre de la gonade que dans celui des anomalies morphologiques. II est donc possible de concevoir une sélection pour ces deux critères.
\end{abstract}

photopérlodisme / descendance / diamètre testiculaire / anomalle morphologique / bélier

Summary - Father-male offspring transmission of seasonal variations in testis diameter and percentage of abnormal spermatozoa in the lle-de-France ram. 1. Male offspring born in February. An experiment was conducted on the lle-de-France (IF) breed to determine if the more or less important sensitivity of the ram to photoperiodism came under genetic control. Five base breed unrelated rams ( 24 yr old), were chosen for this study: 2 good (I and II), 2 bad (III and IV), and an intermediate sire (V). Ram I, which died a few years before the experiment began, was selected on both the low amplitude of its sperm production during a 6-month period of artificial lighting and the very high fertilizing capacity of its sperm in spring. Rams II to $V$ were controlled for 12 (percentage of morphologically abnormal spermatozoa, AM) or 14 (maximum antero-posterior scrotal diameter of

\footnotetext{
- Cette étude fait suite à une publication de 1988 (Reprod Nutr Dev 28, 589-601).
}

** Correspondance et tirés à part. 
both testes, DT) consecutive months. Following this period of control, seasonal variation in rams was assessed as follows: low (ram II), high (rams III and IV) and intermediate (ram V) seasonal variation rams. Breeding (artificial insemination) of these 5 rams to IF ewes resulted in 18 male offspring bom in February and distributed as follows: rams I to III: 4 male offspring/ram (families 1 to $3, F_{1}$ to $F_{3}$ ), rams IV and $V: 3$ male offspring/ram (families 4 and $5, F_{4}$ and $F_{5}$ ). The 18 animals were controlled once a week (DT and AM) from 8,5 to 46 months of age (7 periods, $P_{1}$ to $P_{7}$ ). Regarding DT, mean DT was higher in $F_{1}$ and $F_{2}$ than those of the whole population (WP) (represented by a discontinuous line of ordinate 5.0 in Graph 3), but $F_{2}$ was closer to this population than $F_{1}$. On the other hand, they were lower in the rams of $F_{3}$ and $F_{4}$. Differences between $F_{3}$ or $F_{4}$ and WP were lower in autumn than in spring. All families showed significant differences during the experimental periods except at $P_{7}$ for pairs 3-5 and 4-5 (table I). Distances between $F_{1}, F_{2}, F_{3}$ were always different $(P<0.01$ or $P<$ $0.001)$, whatever the size of the population $(n=3$ or 4$)$. Weekly $F_{5}$ values varied in an opposite way to those of $F_{3}$ and $F_{4}$ : increase in spring and decrease in autumn. Regarding $A M$, families did not differ as much as in DT (graph 4). Weekly variations in AM were also stronger. However, mean AM was almost always lower in $F_{1}$ males and somewhat higher in $F_{3}$ males to that of WP. They fluctuate around the general mean in the $F_{2}, F_{4}$ and $F_{5}$ animals except during periods 1 and 2 where values reached by $F_{5}$ animals were somewhat lower than those of WP. Comparisons of distances between pairs of families (table II) showed that distances were larger in spring $\left(P_{2}, P_{4}, P_{6}\right)$ than in autumn $\left(P_{1}\right.$, $\left.P_{3}, P_{5}, P_{7}\right)$. Pairs 1-3 and 2-3 showed the most difference whatever the number of animals per family. From these data we can conclude that in the IF spring born rams, seasonal variations in DT come under genetic control. These variations are also transmitted in the case of AM to the progeny but with less intensity. Using these criteria, it is thus possible to select rams according to their photosensitivity.

photoperiodicity / progeny / testis diameter / morphological abnormality / ram

\section{INTRODUCTION}

En dépit des nombreuses études consacrées à la reproduction du mâle chez les ovins, on ne sait pas si, pour des individus d'une même race, les différences observées dans les variations saisonnières d'activité sexuelle sont sous contrôle génétique.

Si on connaît l'influence du photopériodisme sur la fonction testiculaire (Ortavant, Thibaut, 1956; Ortavant, 1958; Pelletier, Ortavant, 1975; Alberio, 1976; Lincoln, 1976, 1979; Schanbacher, Ford, 1979, etc), on a du mal à expliquer pourquoi des béliers soumis au même environnement lumineux ont des capacités de reproduction aussi diverses (Islam, Land, 1977; Amir et al, 1986; Colas et al, 1986). II nous est donc apparu utile de rechercher si une telle diversité, qui traduit une différence de sensibilité à la durée d'éclairement, peut se transmettre à la descendance.

Pour caractériser cette sensibilité, nous avons eu recours à la mesure du diamètre antéro-postérieur du scrotum $(D T)$ et du pourcentage de spermatozoïdes anormaux $(A M)$, plus directement liée à la fonction gamétique et d'un emploi plus aisé que les critères endocriniens. La race lle-deFrance a été choisie en raison de l'amplitude des fluctuations de ces deux critères au cours de l'année (Colas et al, 1986).

\section{MATÉRIEL ET MÉTHODES}

Dans une expérience précédente (Colas et al, 1988), nous avons étudié les variations saisonnières du $D T$ et des $A M$ chez 18 descendants de 5 béliers pendant 38 mois (octobre 1980décembre 1983). La présente analyse part des mêmes données regroupées maintenant en 5 familles. 


\section{Animaux expérimentaux}

Le but de cette étude étant de comparer les performances des fils à celles de leur père, nous avons choisi des fondateurs aussi différents que possible : 2 bons reproducteurs (I et II), 2 mauvais (III et IV), et 1 animal intermédiaire (V).

Le premier géniteur (I), mort depuis plusieurs années lorsqu'a débuté l'expérience, faisait partie d'une étude dans laquelle nous n'avions procédé qu'à la mesure de la production spermatique (4 ejac/semaine) pendant une période de 6 mois (durée d'éclairement artificiel variant de 8 à $16 \mathrm{~h}$ en 3 mois et de 16 à $8 \mathrm{~h}$ au cours des 3 mois suivants). Dans le but de contrôler, avec plus de précision, la quantité de spermatozoïdes éjaculés (dont on sait qu'elle est étroitement corrélée aux dimensions de la gonade (Colyer, 1971; Alberio, 1976; Cameron et al, 1988), nous avions fait précéder ce cycle expérimental d'une période d'entraînement (4 éjac/bélier/semaine) de même durée. Ce bélier a été retenu pour ses très bonnes performances : faibles variations "saisonnières" de sa production spermatique (fig 1), pouvoir fécondant élevé au printemps et donc $A M$ peu nombreuses (Colas, 1981).

Les 4 autres fondateurs ont été choisis d'après l'importance des variations saisonnières des deux critères étudiés (DT, $A M$ ) : variations de faible amplitude chez le mâle II, de forte amplitude chez les mâles III et IV, d'amplitude intermédiaire chez le mâle V (fig 2).

Les données de base ont été enregistrées chez les béliers II à IV par les mêmes personnes (une par paramètre).

Les 5 fondateurs sont des animaux d'âge $\geq 4$ ans au début de l'expérience, non apparentés, inscrits à l'UPRA lle-de-France. Leurs descendants $(n=18)$, de même race, ont été extraits d'un lot de mâles nés en février, obtenus par insémination artificelle à partir de la semence de ces 5 géniteurs. Les 18 béliers ont été triés au moment du sevrage (100 j), essentiellement en fonction de la qualité de leurs aplombs et de manière que le poids corporel des 5 familles ainsi constituées soit aussi proche que possible. Le jour des premières collectes de sperme, le poids corporel de chacune d'elle variait de 55,5 a 57,7 $\mathrm{kg}$. Les mères de ces agneaux, différentes pour chacun d'eux, n'avaient d'ascendant commun ni au niveau des parents ni au niveau des grandsparents. Celles-ci appartenaient à 5 lignées qui en aucun cas n'avaient été sélectionnées sur des critères de fécondité.

Les familles 1 à $3\left(F_{1}\right.$ à $\left.F_{3}\right)$, issues des béliers 1 à III, sont composées de 4 descendants, les 2 autres $\left(F_{4}\right.$ et $\left.F_{5}\right)$ n'ont que 3 descendants.

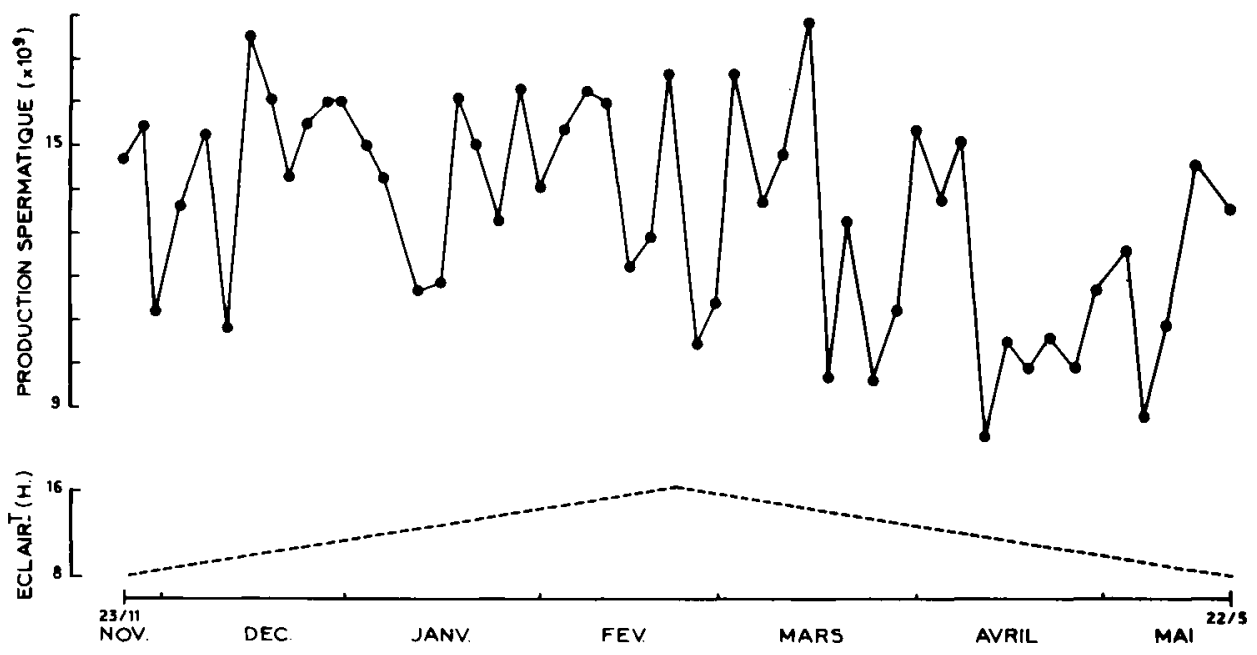

Flg 1. Spermatozoïdes recueillis par séance de collecte (2 éjaculats/séance) chez le bélier fondateur $n^{\circ} 1$ (4 éjaculats/séance) soumis à un cycle lumineux semestriel. 

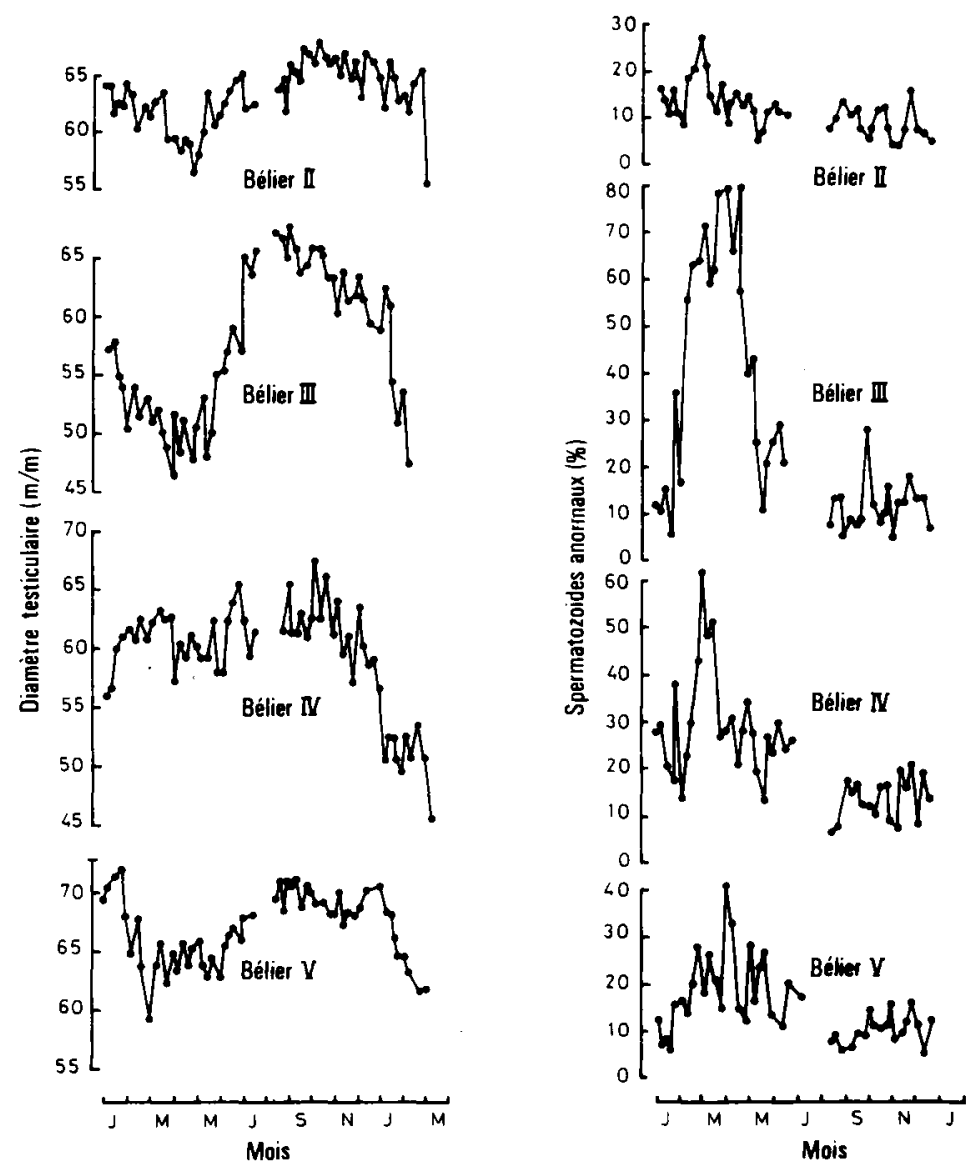

Flg 2. Variations saisonnières du diamètre testiculaire et du pourcentage de spermatozoïdes anormaux chez les 4 béliers fondateurs (II, III, IV, V) exposés à un éclairement naturel.

\section{Contrôles}

La diamètre antéro-postérieur maximum (DT) des testicules (gonade + enveloppe) a été mesuré au $\mathrm{mm}$ près toutes les semaines ( 7 périodes) à l'aide d'un pied à coulisse.

Le pourcentage d'anomalies morphologiques n'étant pas lié au rang de l'éjaculat (Colas, résultats non publiés), nous nous sommes limités à un prélèvement de semence par semaine, le même jour sur tous les animaux, de février à mai ou juin (printemps) et d'août, septembre ou octobre à novembre ou décembre (automne). Ces époques sont appelées "périodes" dans notre étude. Elles sont désignées par la lettre $P$ affectée d'un indice, pair au printemps, impair en automne.

Les spermatozoïdes anormaux (têtes anormales ou sans flagelle, gouttelettes cytoplasmiques en position proximale ou distale, flagelles anormaux) ont été recensés toutes les semaines sur un échantillon de 150 cellules par éjaculat (Colas, 1980). 
Les 18 béliers ont été pesés une fois par mois pendant les 7 périodes expérimentales. Les poids enregistrés ont été interpolés pour les semaines intermédiaires.

\section{Expression et analyse des résultats}

\section{Expression des résultats en variables centrées réduites}

Les variations saisonnières des moyennes du $D T$ et des $A M$ par famille ont été exprimées en notes ou variables centrées réduites selon la formule :

$$
N=[(x-\bar{x}) / \sigma]+5
$$

dans laquelle, $x=$ moyenne hebdomadaire du $D T$ (ou des $A M$ ) d'une famille; $\bar{x}=$ moyenne générale hebdomadaire du même paramètre; $\sigma=$ écart type des moyennes hebdomadaires (figs 3 et 4).

\section{Analyse discriminante}

Les figures 3 et 4 ne donnent aucun élément concernant la variabilité intrafamille du paramètre considéré ( $A M$ ou $D T$ ). Il aurait été possible évidemment, pour chaque semaine et pour chaque couple de famille, de calculer le test classique de différences des moyennes. Mais ce qui importe c'est d'obtenir une réponse pour l'ensemble des mesures d'une même période. D'ou la nécessité de recourir à une analyse plus synthétique des différences entre familles par période. Dans cette analyse, chaque famille a été considérée à chaque période comme une unité et les valeurs d'un même critère ( $D T$ puis $A M$, pour les 3 ou 4 individus qui le composent, comme différentes variables (Lefebvre, 1976).

Le nombre de celles-ci devant être le même pour chaque famille, deux analyses discriminantes, selon la méthode de Rao (1952) ont été réalisées, la première avec les 5 familles de 3 individus, la deuxième avec les 3 familles de 4 individus.

\section{RÉSULTATS}

\section{Allure générale de la courbe}

\section{Diamètre testiculaire}

En dehors de quelques points en $\mathrm{P}_{7}$ pour $F_{2}$ (fig 3 ), les 2 premières familles se situent toujours au-dessus de la moyenne générale (ordonnée 5,0 ). $F_{1}$ est plus éloignée que $F_{2}$ de cette moyenne et à une distance quasi constante de celle-ci (environ un écart type).

$F_{3}$ et $F_{4}$ ont des valeurs inférieures à la moyenne de la population. Leur évolution au cours des 7 périodes est plus irrégulière : les moyennes testiculaires se rapprochent de l'axe en automne $\left(\mathrm{P}_{3}, \mathrm{P}_{5}, \mathrm{P}_{7}\right)$ et s'en écartent au printemps $\left(P_{2}, P_{4}, P_{6}\right)$, en particulier en mars-avril. Pendant cette saison, $F_{4}$ a plus tendance à s'éloigner de la moyenne que $F_{3}$.

Dès le départ $\left(P_{1}\right)$, les 4 familles $\left(F_{1}, F_{2}\right.$, $F_{3}, F_{4}$ ) adoptent une position par rapport à la moyenne et un classement qu'elles conserveront ultérieurement $\left(P_{2}\right.$ à $\left.P_{7}\right)$.

L'évolution du DT de la dernière famille $\left(F_{5}\right)$ est inverse de celle des familles 3 et 4: augmentation des valeurs au printemps, diminution en automne. Celles-ci restent dans l'ensemble inférieures à la moyenne.

\section{Anomalies morphologiques}

Les différences entre familles (fig 4) sont moins accusées et les variations hebdo- 


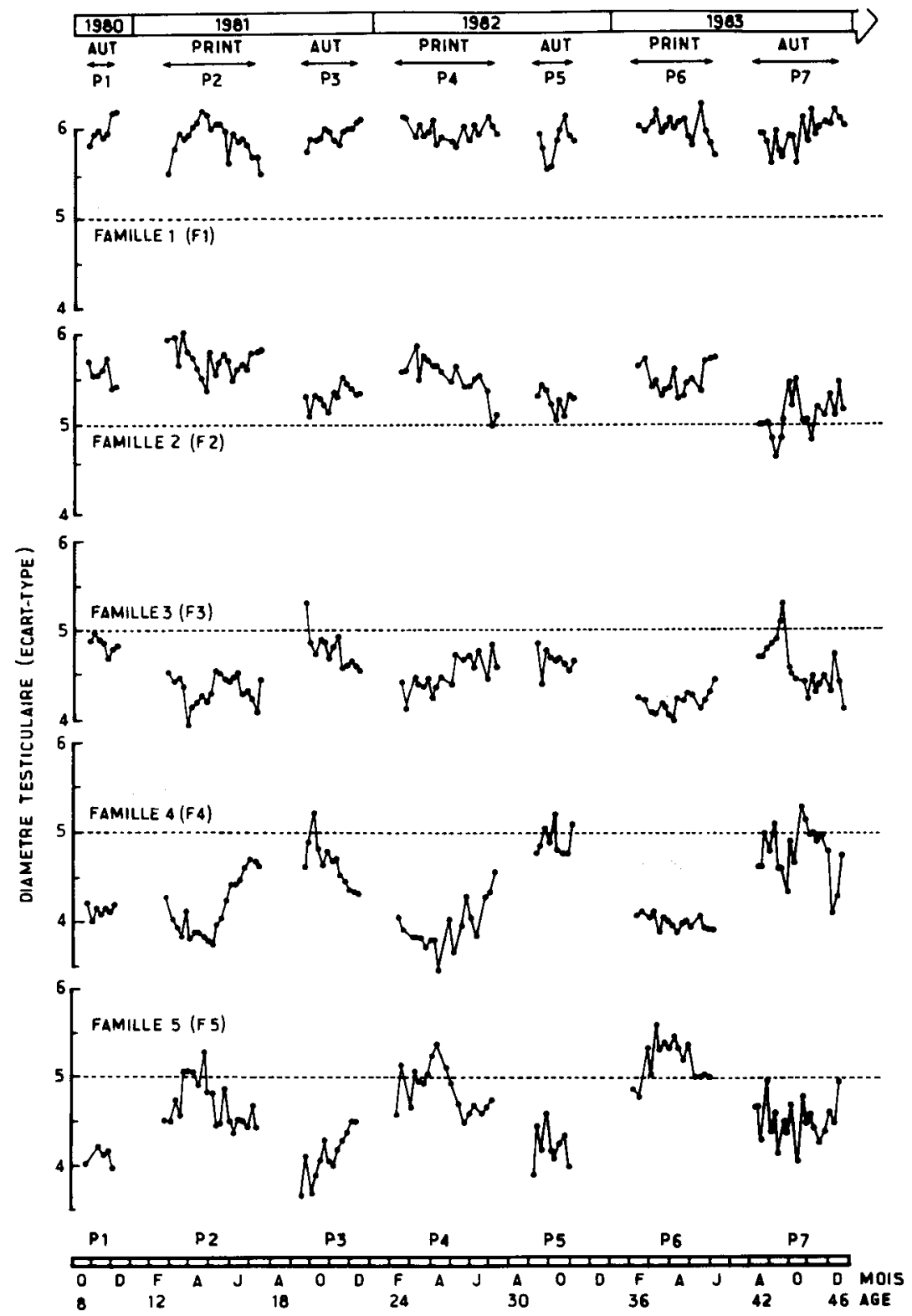

Flg 3. Variations saisonnières (exprimées en variables centrées réduites) du diamètre testiculaire chez les 5 familles issues des 5 béliers fondateurs $\left(4\right.$ individus par famille pour $F_{1}, F_{2}, F_{3} ; 3$ individus pour $F_{4}$ et $F_{5}$ ). 


\begin{tabular}{|c|c|c|c|c|c|c|}
\hline 1980 & \multicolumn{2}{|c|}{1981} & \multicolumn{2}{|c|}{1982} & \multicolumn{2}{|c|}{1983} \\
\hline AUT & PRINT & AUT & PRINT & AUT & PRINT & $\triangle Q T$ \\
\hline PI & $\mathbf{P}_{2}$ & P3 & $P_{4}$ & P5 & P5 & P7 \\
\hline
\end{tabular}

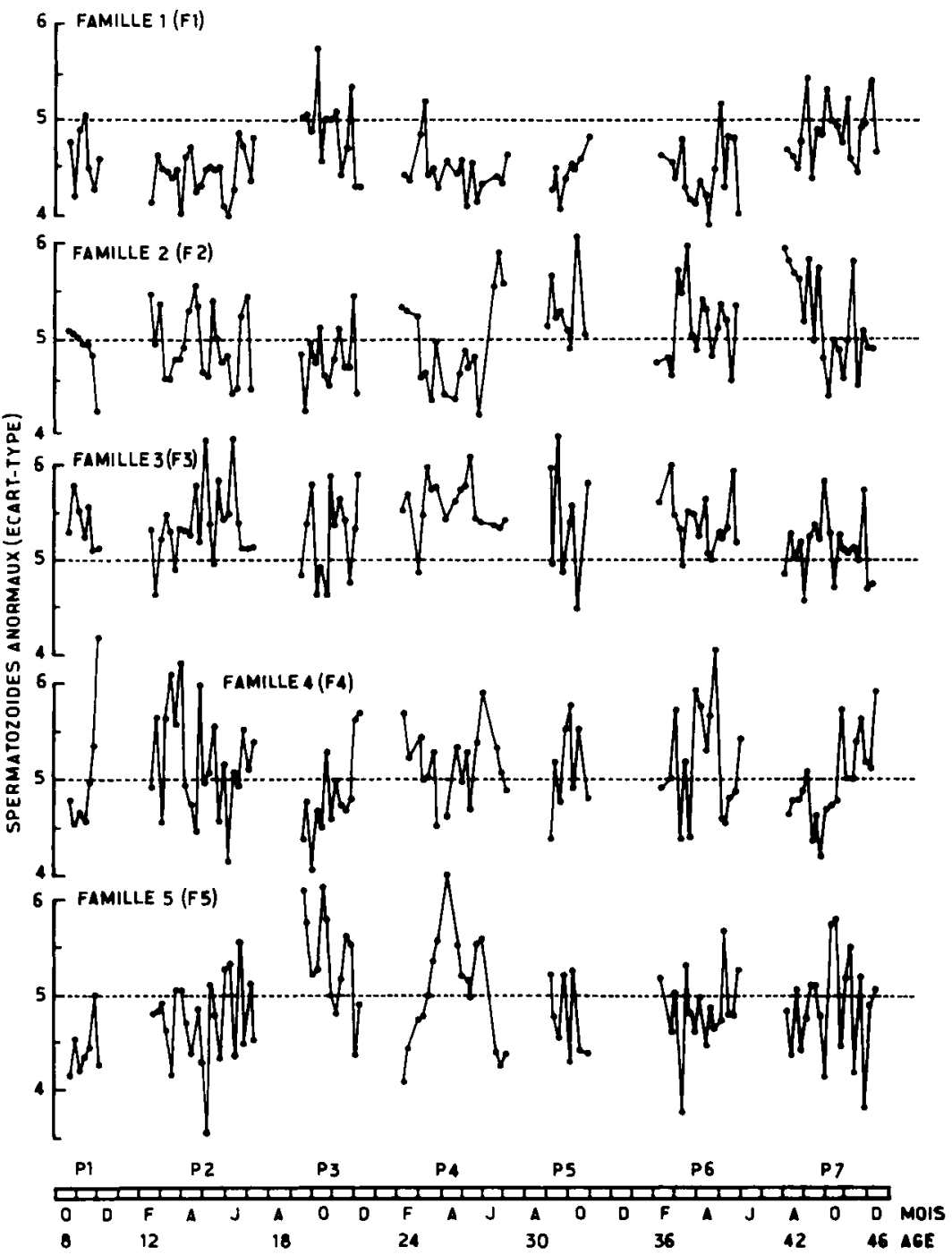

Fig 4. Variations saisonnières (exprimées en variables centrées rédslites) du pourcentage de spermatozoïdes anormaux chez les 5 familles issues des 5 béliers fondateurs (4 individus par famille pour $F_{1}, F_{2}, F_{3} ; 3$ individus pour $F_{4}$ el $F_{5}$ ). 
madaires plus fortes que dans le cas précédent. On voit cependant que les courbes représentatives des 7 périodes se situent presque toujours au-dessous de la moyenne générale chez les individus de la famille 1 et au-dessus chez ceux de la famille 3 , en particulier au printemps et pendant la 1re période. Celles des familles 2 et 4 se situent toutes deux autour de la moyenne, sans que l'on puisse déceler de tendance particulière pour l'une d'elles au printemps. Les valeurs enregistrées sur la famille 5 sont égales ou inférieures à la moyenne en $P_{1}$ et $P_{2}$. Elles fluctuent autour de la moyenne ensuite.

\section{Comparaison des distances entre familles selon les périodes. Étude des tests statistiques}

Les comparaisons ont été faites, pour les deux paramètres $A M$ et $D T$, à partir des seuils de signification des tests $F$ et non des distances elles-mêmes $\left(D^{2}\right)$ en raison de la variation importante du nombre de degrés de liberté selon les périodes. Nous avons représenté, à titre d'exemple, la projection des 5 familles, à la $6^{\ominus}$ période, sur le plan des deux premiers axes (fig 5). $\mathrm{Ce}$ type de représentation rend compte de la

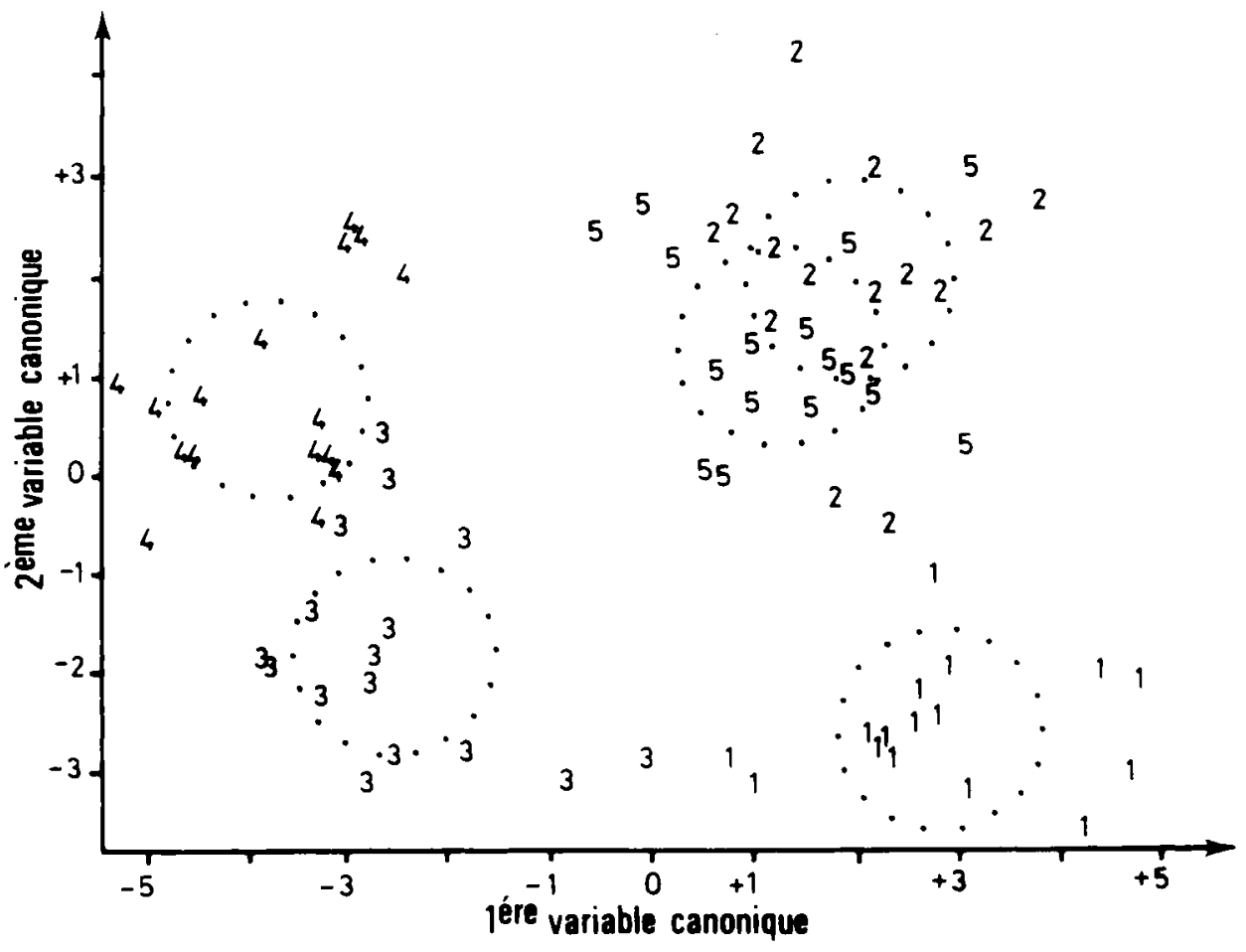

Fig 5. Discrimination des 5 familles pendant la $6^{\theta}$ période en fonction du diamètre testiculaire de leurs 3 éléments (béliers). (Pour des raisons de clarté, les 5 familles ont été désignées par leur chiffre respectif 1 à 5). Les cercles sont les projections des sphères de dispersion de rayon unité, données par l'analyse discriminante. 
dispersion des données autour de la moyenne, dispersion qui n'apparaît pas sur un graphique exprimé en variables centrées réduites.

\section{Diamètre testiculaire}

Les 5 familles sont significativement différentes pendant toute la durée de l'expérience, sauf à la $7^{\mathrm{e}}$ période pour les couples 3-5 et 4-5 (tableau I), ce qui confirme les résultats énoncés dans le paragraphe précédent. Les résultats sont identiques lorsque le $D T$ est divisé par le poids, sauf à la 7 e période où seules les familles 2 et 5 ne sont pas significativement différentes.

Les distances entre les 3 premières familles sont toujours hautement ou très hautement significatives, et ce quel que soit le nombre d'individus ( 3 ou 4) par famille (tableau I).

C'est entre les familles 3 et 4 que les valeurs de $F$ sont les plus faibles.

Sur l'exemple choisi ( $6^{\theta}$ période, fig 5) on voit que :

- les familles 2 et 5 d'une part, 3 et 4 d'autre part sont les moins éloignées (mais leur distance reste significative),

- les couples 1-2 et 3-4 sont très éloignés.

Sur ce graphique, on retrouve donc sous une forme plus détaillée les résultats déjà exprimés sur le tableau I (pour $\mathrm{P}_{6}$ ).

\section{Anomalies morphologiques}

Les valeurs des tests $F$ et les seuils de signification (tableau II) sont beaucoup plus faibles que ceux du tableau précédent. On voit néanmoins qu'il y a plus de différence entre familles au printemps $\left(P_{2}, P_{4}, P_{6}\right)$ qu'en automne, et en particulier qu'en $P 1$, où l'on ne relève qu'une seule valeur de $F$ significative $\left(3,5^{*}\right.$, couple $\left.3-4\right)$.
C'est aussi entre les familles 1 et 3 et les familles 2 et 3 qu'on rencontre, dans l'ensemble, le plus de différences. Enfin, les résultats des comparaisons établies à partir de 3 ou bien de 4 mâles par famille sont concordants. Les valeurs de $F$ sont d'ailleurs très proches.

\section{DISCUSSION}

Cette étude a permis de mettre en évidence des différences significatives dans l'évolution des dimensions testiculaires et de la qualité des gamètes entre les 5 familles, et ce malgré le faible effectif animal utilisé (3-4 par famille).

\section{Diamètre testiculaire}

Les résultats enregistrés chez les fils vont bien dans le même sens que les observations faites sur les pères. Au printemps $\left(P_{2}, P_{4}, P_{6}\right)$ comme en automne $\left(P_{3}, P_{5}\right.$, $P_{7}$ ), chaque famille conserve la même position par rapport à la moyenne générale. Ces résultats permettent de classer les 5 familles et leur fondateur dans un ordre à peu près équivalent : respectivement $F_{1}$, $F_{2}, F_{5}, F_{3}, F_{4}$ et I, II, V, IV, III. On voit en effet que les mâles I et II, considérés comme de bons reproducteurs, peu sensibles au photopériodisme, ont des descendants supérieurs à la moyenne de la population, dont les testicules n'involuent pas au printemps. Les béliers III et IV au contraire connaissent à la même saison une forte régression gonadique qui apparaît également chez leurs fils. L'écart est même spectaculaire lorsqu'on compare les groupes les plus éloignés $F_{1}$ et $F_{4}$ (plus de 2 écarts types au printemps).

On peut noter aussi que les seuils de signification restent à peu près inchangés 


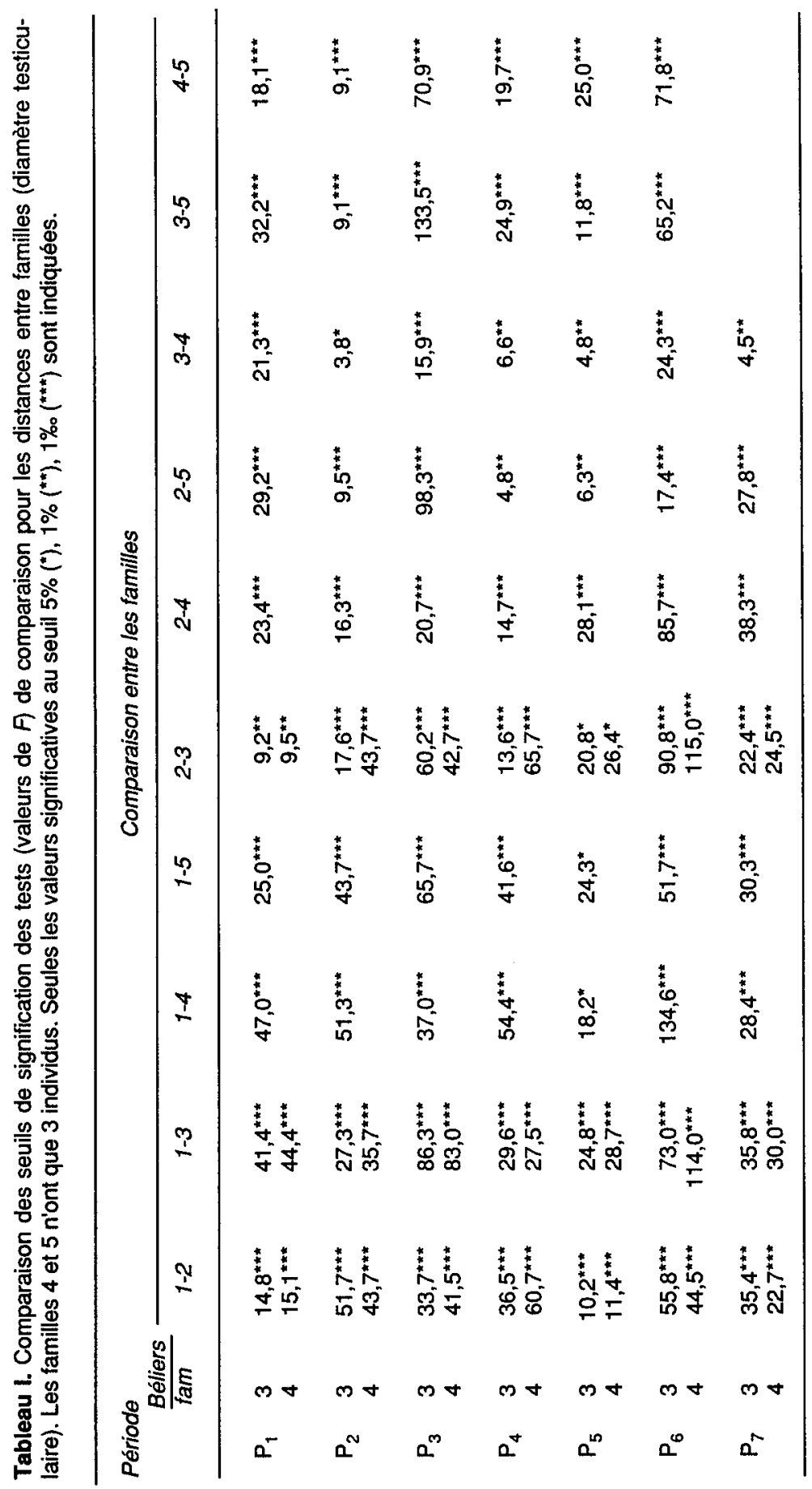


Photosensibilité, liaison père-fils

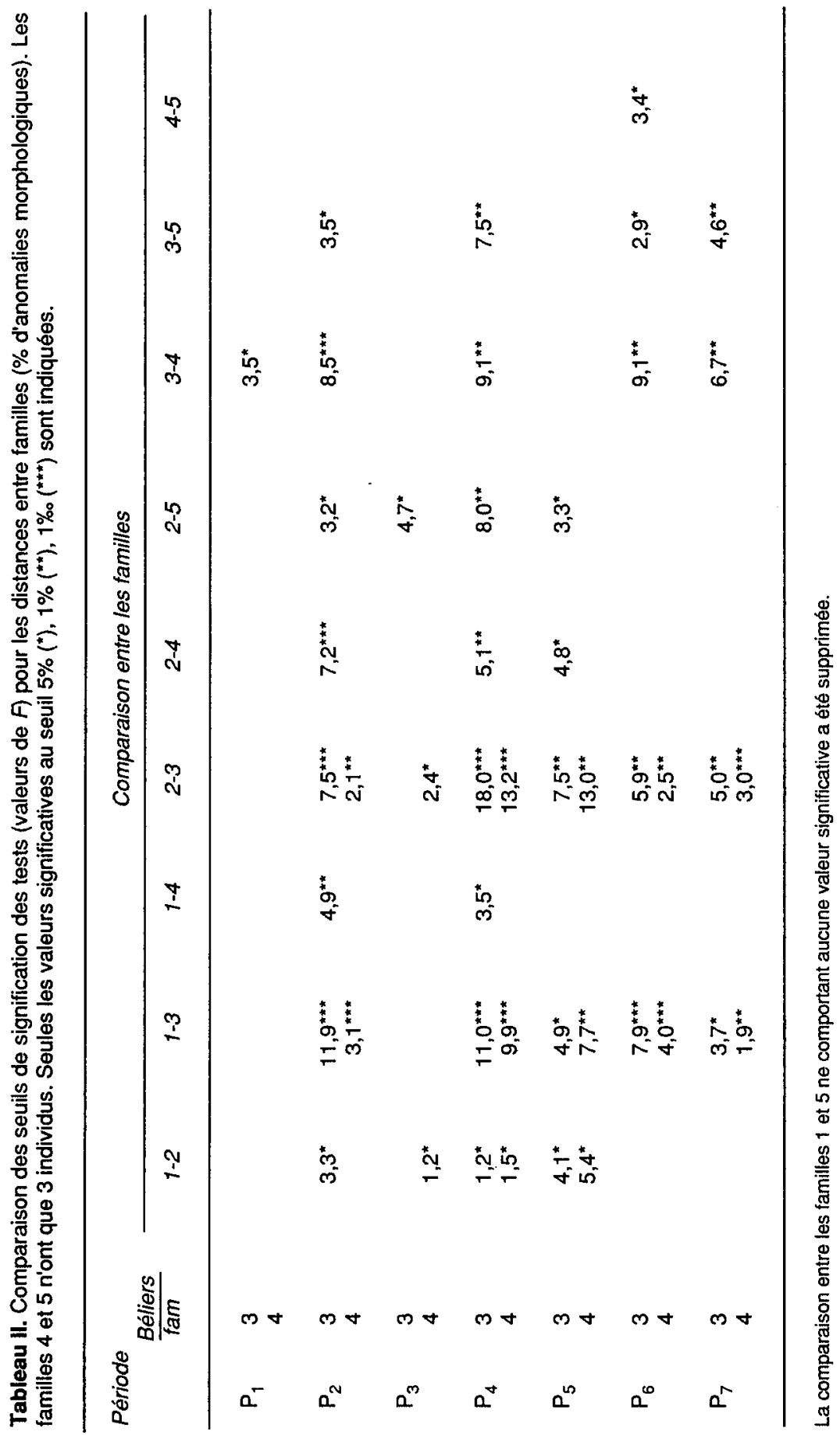


lorsqu'on passe de 3 à 4 individus par groupe $\left(F_{1}, F_{2}, F_{3}\right)$, ce qui traduit une assez bonne homogénéité intrafamille pour le caractère étudié.

$\mathrm{Au}$ printemps, les animaux du $5^{\mathrm{e}}$ groupe ont des dimensions scrotales comparables à celles de la moyenne générale. À cette saison, ils sont donc, comme leur fondateur, intermédiaires entre les 2 meilleures familles $\left(F_{1}, F_{2}\right)$ et les 2 plus mauvaises $\left(F_{3}, F_{4}\right)$. Toutefois, la régression testiculaire observée en automne est surprenante. Elle peut difficilement s'expliquer par l'ascendance maternelle des béliers (non apparentés), tous issus de mère différente. La connaissance de la production spermatique (au moins 4 éjaculats par bélier et par semaine (Colas, Laszczka, non publié) n'apporterait pas ici d'élément nouveau, les deux caractères étant hautement corrélés en particulier chez cette race (Alberio, 1976). On pourrait penser aussi à la mesure des niveaux endocriniens ( $F S H$ et $L H$ ). On sait en effet que la croissance testiculaire est toujours précédée d'une stimulation de l'axe hypothalamo-hypophysaire et de la sécrétion des hormones gonadotropes (Pelletier, Ortavant, 1970; Courot, Ortavant, 1981). En réalité, cette mesure ne ferait que confirmer l'évolution de la gonade. En l'état actuel de nos connaissances, aucune explication plausible ne peut donc être avancée.

Les données rapportées dans notre étude permettent de penser que la sensibilité du bélier au photopériodisme, telle que nous l'avons définie, est un caractère génétique. Son expression dans la descendance reflète le degré d'intensité du phénomène paternel. Nos résultats sont à rapprocher de ceux de Lincoln (1989) à propos des races Soay et Portland, dont le croisement engendre, chez le mâle, des individus intermédiaires entre les génotypes parentaux. La mesure de cette sen- sibilité pourrait être à la base d'une sélection des reproducteurs chez les races les plus affectées par le photopériodisme. On conçoit en effet l'intérêt d'identifier et de multiplier les géniteurs de type I et II, capables de se reproduire au printemps, sans traitement photopériodique et d'éliminer, ou de soumettre à un conditionnement lumineux, ceux dont la fonction sexuelle s'effondre au printemps et qui semblent ne pouvoir engendrer que des fils à leur image. Toutefois, dans le cas de la race lle-de-france, les naissances ont généralement lieu en octobre-novembre, c'est-àdire à une époque différente de celle de nos béliers. Aussi faut-il attendre la suite de cette étude, réalisée sur des mâles nés en automne (actuellement en cours de rédaction), pour savoir si les conclusions énoncées peuvent être généralisées.

Dans une étude précédente (Colas et al, 1988), nous avions montré qu'il existe une relation étroite entre les données relevées en avril sur des béliers antenais et celles que l'on enregistre 12 à 24 mois plus tard sur les mêmes individus (exemple : $\rho^{2}=0,69$ entre $P_{2}$ et $P_{4}, 0,88$ entre $P_{2}$ et $P_{6}$ ) (Colas et al, 1988). Les corrélations étant identiques en automne, il suffit d'effectuer des contrôles pendant deux saisons consécutives (printemps et automne) pour connaître la sensibilité d'un mâle à son environnement lumineux. Lorsqu'elle est toujours effectuée à la même époque de l'année, la mesure du diamètre testiculaire antéro-postérieur est donc une mesure fiable, c'est-à-dire répétable et héritable (Lee, Land, 1985; Ricordeau et al, 1986). D'autres auteurs sont parvenus à la même conclusion à propos de la circonférence scrotale (Langford et al, 1989).

II aurait été intéressant de savoir si les filles des 5 béliers fondateurs présentaient des différences dans leur durée d'anœstrus, que l'on sait très liée à l'envi- 
ronnement lumineux chez les ovins (Thimonier et Mauléon, 1969; Ortavant et al, 1988; Thimonier, 1989). On peut en effet établir un certain parallélisme entre les 2 sexes : comme la fonction testiculaire, la fonction ovarienne dépend du photopériodisme (Thimonier, Mauléon, 1969; Thimonier, 1989) et la longueur de la période ovulatoire varie selon la race et l'individu (Wheeler, Land, 1977; Thimonier, 1989). Par ailleurs, il est admis que de faibles oscillations saisonnières des dimensions de la gonade vont généralement de pair avec une courte durée d'anœestrus. C'est ce que l'on constate en comparant la race Préalpes aux races lle-de-France (Thimonier et Mauléon, 1969; Pelletier, comm pers) et Merinos (Wheeler, Land, 1977). If n'est donc pas impossible qu'il existe chez la femelle une composante génétique dans l'expression de sa sensibilité à la lumière. En raison de son importance zootechnique, ce problème mériterait d'être abordé.

\section{Anomalies morphologiques}

L'étude de la morphologie cellulaire n'a pas permis de mettre en évidence des différences entre lignées aussi prononcées que celles que nous avons constatées pour le diamètre testiculaire. Comme on pouvait s'y attendre, ces différences sont plus fréquentes au printemps qu'en automne. Seules 2 familles s'opposent vraiment : la famille 1 et la famille 3 . Les béliers I et III sont donc ceux qui ont engendré les descendants les plus différents par leur production spermatique (liée étroitement aux dimensions testiculaires) (Colyer, 1971; Alberio, 1976; Cameron et al, 1988) et par la qualité de leurs gamètes. Quant à la famille 2, le tableau II montre qu'elle est tout de même supérieure à la famille 3 . Au total, les écarts qui existaient entre les géniteurs I, II et III se retrouvent aussi chez leurs fils mais sous une forme plus atténuée. La mesure du pourcentage de formes anormales est donc un critère moins sélectif et moins précis que le critère testiculaire. II n'est bénéfique au niveau de la descendance que pour des producteurs de très bonne ou de très mauvaise semence. Cette moindre efficacité tient au fait que la répétabilité des examens qualitatifs est plus faible chez un même individu, que celle des dimensions gonadiques (Colas et al, 1988). Elle indique aussi que le nombre et la qualité des spermatozoïdes produits n'évoluent pas toujours dans le même sens. II est donc important de pouvoir déceler les béliers porteurs des deux caractères. Une telle sélection ne peut évidemment être menée à bien qu'après un contrôle minutieux (un éjaculat par semaine) des animaux pendant les périodes critiques de l'année, c'est-à-dire de mars à mai et en septembre. II n'est pas impossible, d'ailleurs, que la seule prise en compte des anomalies plus directement liées à la photopériode (gouttelettes proximales) (Colas, 1980) permette d'augmenter la précision de la sélection.

\section{CONCLUSION}

Chez les mâles lle-de-France nés au printemps, les variations saisonnières du diamètre antéro-postérieur du testicule se transmettent à la descendance. Elles s'expriment également dans le cas des anomalies morphologiques, mais avec moins d'intensité. Ces deux critères sont donc un bon révélateur de l'influence du photopériodisme sur l'activité sexuelle du mâle. Leur examen permet de sélectionner ces individus sur leur plus ou moins grande sensibilité à leur environnement lumineux. 


\section{REMERCIEMENTS}

Les auteurs tiennent à remercier le personnel de la bergerie pour l'aide efficace qu'il leur a apportée au cours de cette expérience.

\section{RÉFÉRENCES}

Alberio R (1976) Rôle de la photopériode dans le développement de la fonction de reproduction chez l'agneau lle-de-France, de la naissance à 21 mois. Thèse Doct $3^{\theta}$ cycle, Univ Paris VI, $57 \mathrm{p}$

Amir D, Gacitua H, Ron M, Lehrer AR (1986) Seasonal variation in semen characteristics and the fertility of Finn cross rams subjected to frequent ejaculations. Anim Reprod Sci $10,75-84$

Cameron AWN, Fairnie IJ, Curnow DH, Keoghe J, Lindsay DR (1988) The output of spermatozoa of naturally mated rams. 10th int Congr Anim Reprod and Al UrbanaChampaign, II, vol D $n^{\circ} 266$

Colas G (1980) Variations saisonnières de la qualité du sperme chez le bélier lle-deFrance. I. Etude de la morphologie cellulaire et de la motilité massale. Reprod Nutr Dev 20, 1789-1799

Colas G (1981) Variations saisonnières de la qualité du sperme chez le bélier lle-deFrance. II. Fécondance : relation avec les critères qualitatifs observés in vitro. Reprod Nutr Dev21, 399-407

Colas G, Guérin Y, Lemaire Y, Montassier Y, Despierres J (1986) Variations saisonnières du diamètre testiculaire et de la morphologie des spermatozoïdes chez le bélier Vendéen et chez le bélier Texel. Reprod Nutr Dev 26, 863-875

Colas G, Paquignon M, Chemineau PH (1986) Environnement et fertilité des mâles chez les petits ruminants et chez les porcins. In: Recherches récentes sur l'épidémiologie de la fertilité. Masson, Paris, 129-141

Colas G, Lefebvre J, Guérin Y (1988) Recherche d'une prévision précoce de l'amplitude des variations saisonnières du diamètre testiculaire et du pourcentage de spermatozoïdes anormaux chez le bélier lle-de-
France. 1. Animaux nés en février. Reprod Nutr Dev 28, 589-601

Colyer $J$ (1971) Development of the testis and epididymis in the Clun Forest ram. J Agric Sci Camb 76, 433-441

Courot M, Ortavant R (1981) Endocrine control of spermatogenesis in the ram. J Reprod Fertil, Suppl 30, 47-60

Islam ABMM, Land RB (1977) Seasonal variation in testis diameter and sperm output of rams of breeds of different prolificacy. Anim Reprod 25, 311-317

Langford GA, Shrestha JNB, Marcus GJ (1989) Repeatability of scrotal size and semen quality measurements in rams in a short-day light regime. Anim Reprod Sci 19, 19-27

Lee GJ, Land RB (1985) Testis size and LH response to LH-RH as male criteria of female reproductive performance. In: Genetics of Reproduction in sheep (Land RB, Robinson DW, eds). Butterworths, London, 333-341

Lefèbvre J (1976) Introduction aux analyses statistiques multidimensionnelles. Masson, Paris, $275 \mathrm{p}$

Lincoln GA (1976) Secretion of LH in rams exposed to two different photoperiods. J Reprod Fertil 47, 351-353

Lincoin GA (1979) Photoperiodic control of seasonal breeding in ram: participation of the cranial sympathetic nervous system. $J$ Endocrinol 82, 135-147

Lincoln GA (1989) Seasonal cycles in testicular activity in Mouflon, Soay sheep and domestical breeds of sheep: breeding seasons modified by domestication. Zool J Linn Soc 95, 137-147

Ortavant $R$ (1958) Le cycle spermatogénétique chez le bélier. Thèse Doct Sci $n^{\circ}$ A 311803990, Paris, $127 p$

Ortavant R, Thibault C (1956) Influence de la durée d'éclairement sur la production spermatique du bélier. CR Séances Soc Biol Fil 150, 358-361

Ortavant R, Bocquier F, Pelletier J, Ravault JP, Thimonier J, Volland-Nail P (1988) Seasonality of reproduction in sheep and its control by photoperiod. Aust J Biol Sci 41, 69-85

Pelletier J, Ortavant R (1970) Influence du photopériodisme sur les activités sexuelle, hypophysaires et hypothalamique du bélier lle-deFrance. In : La Photorégulation de la repro- 
duction chez les oiseaux et chez les mammifères (Benoit J, Assenmacher I, eds). Coll CNRS, Montpellier, 483-495

Pelletier J, Ortavant R (1975) Photoperiodic control of LH release in the ram. I. Influence of increasing and decreasing light photoperiods. Acta Endocrinol 78, 435-441

Rao CR (1952) Advanced Statistical Methods in Biometrics Research. John Wiley, London, New York, $390 p$

Ricordeau G, Poivey JP, Bodin L, Barillet F, Roussely M (1986) Importance of testicular measurements of young males tested on individual performance for improvement of their daughters prolificacy: application to the selection scheme on Lacaune milking breed. 3rd World Cong Genetics Livest Prod XI, 90-95
Schanbacher BD, Ford JJ (1979). Photoperiodic control of ovine spermatogenesis: relationship to serum hormones. Biol Reprod 20, 719-726

Thimonier J (1989) Contrôle photopériodique de l'activité ovulatoire chez la brebis. Existence de rythmes endogènes. Thèse Doct Sci Tours, $112 p$

Thimonier J, Mauléon P (1969) Variations saisonnières du comportement d'œstrus et des activités ovarienne et hypophysaire chez les ovins. Ann Biol Anim Biochim Biophys 9, 233-250

Wheeler AG, Land RB (1977) Seasonal variation in cestrus and ovarian activity of finnish Landrace Tasmanian Merino and Scottish Blackface ewes. Anim Prod 24, 363-376 